\title{
BEM modeling of wave diffraction in an elastic matrix with nano-heterogeneities
}

\author{
S. L. Parvanova ${ }^{1}$, G. D. Manolis ${ }^{2} \&$ P. S. Dineva ${ }^{3}$ \\ ${ }^{1}$ Department of Structural Engineering, \\ University of Architecture, Civil Engineering and Geodesy, Bulgaria \\ ${ }^{2}$ Department of Civil Engineering, Aristotle University, Greece \\ ${ }^{3}$ Institute of Mechanics, Bulgarian Academy of Sciences, Bulgaria
}

\begin{abstract}
This work addresses wave diffraction and scattering in a homogeneous, elastic matrix containing multiple heterogeneities at nano-scale. The source of wave motion is an incident, time harmonic elastic pressure $(\mathrm{P})$ wave propagating through the heterogeneous matrix. The approach followed here is based on a combination of (a) classical elastodynamic theory for the bulk solid, relating the total wave fields with the incident and scattered ones through the superposition principle; (b) non-classical boundary conditions and localized constitutive equations for the matrix-heterogeneity interface in the framework of the GurtinMurdoch surface elasticity theory. The method of solution is the boundary element method using frequency-dependent fundamental solutions for the governing equations of the bulk solid.
\end{abstract}

Keywords: wave diffraction, stress concentration, wave motion, Gurtin-Murdoch model, nano-cavities, nano-inclusions, boundary elements.

\section{Introduction}

In this last decade, nanostructured materials (e.g. nanocrystals, nanochannel arrays, nanocomposites containing coated nanowires, etc.), nanoscale structural elements (e.g. quantum dots, quantum wires, carbon nanotubes, nanorods, thin films, nanoparticles, nanoplates) and finally nanodevices used in telecommunucation networks, plus nano-opto-electro-mechanical systems, nanoprobes and nanosensors have all become increasingly popular because of their unique mechanical, electronic, chemical and optical properties. The fabrication 
and use of these materials and components, however, require novel developments in both theoretical and applied mechanics, as well as new and specialized computational techniques, see Srivastava and Atluri [1].

One of the main thrusts of what is now known as nano-scale mechanics is the so called multi-scale approach, which aims at extending the range of classical continuum mechanics by bridging the basic principles of the continuum mechanics theory with the effects observed at the molecular level. Along this direction, we have the pioneering works of Gurtin and Murdoch [2,3] who developed a general theoretical framework to account for both surface and interface stresses. In their model, the interfaces between the nano-inhomogeneity and the surrounding matrix are regarded as thin membranes that possess their own mechanical properties and surface tension. Then, residual stresses develop around the surface and the interface, even in the absence of external loading. The effect of these residual stresses is size-dependent and their influence becomes very significant for nanosized materials.

Next, the techniques used for solving both static and dynamic problems of solids containing nano-inhomogeneities are as follows: (a) analytical, such as the complex variable technique, see Tian and Rajapakse [4], and the wave function expansion method, see Wang [5], Wang et al. [6] and Ru et al. [7]; (b) finite element method (FEM), see Wang et al. [6]; (c) boundary element method (BEM), see Dong [8], including the complex variable BEM, see Jammes et al. [9]. The conclusions, which can be drawn at this stage are as follows: (a) to date, there is a limited number of theoretical and numerical studies for solids on surface effects as seen from the nano-scale; (b) most of the solution techniques that have been developed are semi-analytical, with relatively few papers using the boundary element approach, which is known to be a highly accurate numerical tool. Also, most BEM solutions are for problems under static conditions; (c) to the best of our knowledge, there are no BEM-generated results for elastic wave diffraction and scattering by nano-inhomogeneities embedded in a solid matrix.

In this work, we develop BEM solutions for dynamic stress concentration phenomena in the vicinity of multiple circular nanoholes and/or nano-inclusions of arbitrary size, geometrical configuration, material properties, and taking into account surface effects at the same time. A systematic study of this kind involving the dynamic response of an infinite solid matrix with multiple heterogeneities acting both as wave scatterers and stress concentrators is, in our opinion, necessary in view of potential technological developments in the field of nanostructures.

Thus, the present work is an effort in this direction and is broken down as follows: Section 2 defines the boundary value problem (BVP) for in-plane wave motion problem, while section 3 presents a formulation starting with a boundary integral equations statement along the interface between the nano-inclusion and the surrounding solid matrix. Next, section 4 presents the results of a rather detailed verification study, while section 5 presents new results from parametric studies that serve to establish the non-uniform stress distribution in the solid matrix as a function of the internal distribution of multiple nano-heterogeneities. Finally, the paper ends with a list of conclusions. 


\section{Problem statement}

We consider the in-plane problem, where an elastic matrix of infinite extent contains multiple cylindrical elastic inclusions of arbitrary size, number, material properties and geometrical configuration and is swept by time-harmonic pressure (P) waves. The $\mathrm{n}$-th interface between the matrix and the $n$-th nano-inclusion is denoted by $\Gamma_{I}^{n}$, where $n=1,2, \ldots N$. . It is assumed that inclusions do not intersect and their total surface is denoted as $\Gamma_{I}=\bigcup_{n=1}^{N} \Gamma_{I}^{n}$. The elastic (or Lame) constants and the density of the matrix are $\lambda_{M}, \mu_{M}, \rho_{M}$, while those of the n-th inclusion are $\lambda_{I}^{n}, \mu_{I}^{n}, \rho_{I}^{n}$. A Cartesian coordinate system $O x_{1} x_{2} x_{3}$ is next introduced and inplane wave motion is studied with respect to the plane $x_{3}=0$. Furthermore, it is assumed that all field quantities are time harmonic with frequency $\omega$ of the incident wave, so that the common factor $\exp (\iota \omega t)$ in all dependent variables can be omitted. Once the incident ('in') wave impinges on the inclusions, a scattered ('sc') wave is generated and the total wave field is given by superposition as $u_{i}=u_{i}^{i n}+u_{i}^{s c}$ and $t_{i}=t_{i}^{i n}+t_{i}^{s c}$ for the displacements and tractions, respectively. Next, the displacement that develops at field point $\left(x_{1}, x_{2}\right)$ once incident P-wave has propagated past it has the following form: $\left(\begin{array}{c}u_{1}^{\text {in }} \\ u_{2}^{\text {in }}\end{array}\right)=u_{0 P}\left(\begin{array}{c}\cos \theta \\ \sin \theta\end{array}\right) \exp \left[-i k_{P}\left(x_{1} \cos \theta+x_{2} \sin \theta\right)\right]$, where $u_{0 P}$ is the unit displacement amplitude, $k_{P}=\omega / C_{P}, C_{P}=\sqrt{\left(\lambda_{M}+2 \mu_{M}\right) / \rho_{M}}$ respectively are the wave number and the phase velocity of the wave, and $\theta$ is the incident angle between the direction of propagation and axis $O x_{1}$.

For the problem defined above, the only nonzero quantities are the two displacement vector components $u_{i}\left(x_{1}, x_{2}, \omega\right), i=1,2$, the stresses $\sigma_{i j}\left(x_{1}, x_{2}, \omega\right), i, j=1,2$ plus the corresponding traction vector components $t_{i}\left(x_{1}, x_{2}, \omega\right)=\sigma_{i j}\left(x_{1}, x_{2}, \omega\right) n_{j}$, where $n_{i}$ is the outward pointing, unit normal vector. In the bulk solid, the equations of motion in the absence of body forces is

$$
\sigma_{i j, i}+\rho \omega^{2} u_{j}=0
$$

where $\sigma_{i j}=\left\{\begin{array}{c}C_{i j k l}^{M} u_{k, l}^{M} \text { in the matrix } \\ C_{i k k l}^{I, n} u_{k, l}^{I, n} \text { in the } n-\text { th inclusion }\end{array}, C_{i j k l}=\lambda \delta_{i j} \delta_{k l}+\mu\left(\delta_{i k} \delta_{j l}+\delta_{i l} \delta_{j k}\right)\right.$.

Here, commas subscripts denote partial differentiation with respect to the spatial coordinates and the summation convention over repeated indices is implied. 
The classical theory of elasticity is applicable to the bulk solid, but on the various surfaces, the interface stresses which develop lead to a set of non-classical boundary conditions. At first, assume that the $n$-th interface between each nanoinclusion and its surrounding matrix is regarded as a thin material surface that possesses its own mechanical properties $\lambda^{S, n}, \mu^{S, n}$ and surface tension $\tau^{0, n}$. More specifically, $\tau^{0, n}$ is the residual surface tension under unstrained condition that will induce an additional static deformation, but in dynamic analysis this is often ignored. These interfacial effects are described in the theory developed by Gurtin and Murdoch [2,3] and Gurtin et al. [10]. Following their model, the constitutive equation along the $j$-th interface $\Gamma_{i}^{j}$ between the solid matrix and $j$-th inclusion is written as

$$
\sigma^{s u r, j}=\tau_{j}^{0}+\left(2 \mu_{j}^{S}+\lambda_{j}^{S}\right) \varepsilon^{s u r, j}
$$

In the above, $\sigma^{s u r, j}, \varepsilon^{s u r, j}$ respectively are the stress and the corresponding strain along interface $\Gamma_{i}^{j}$. Next, the boundary conditions along $\Gamma_{i}^{j}$ are as follows [11]:

(a) Continuity of displacements along the $j$-th interface coming from the side of the heterogeneity $u_{i}^{j}$ and from the side of the surrounding matrix $u_{i}^{M}$ in the $O x_{1} x_{2} x_{3}$ coordinate system:

$$
u_{i}^{j}=u_{i}^{M} ; i=1,2
$$

(b) Interface equilibrium conditions along an arc length $s_{j}$, i.e. on the undeformed interface $s_{j} \equiv \Gamma_{i}^{j}$ in terms of the local normal and tangential $(n, t)$ coordinates defined on $s_{j} \equiv \Gamma_{i}^{j}$ :

$$
\begin{gathered}
\left\{\begin{array}{l}
\sigma_{n}^{j}-\sigma_{n}^{M} \\
\sigma_{t}^{j}-\sigma_{t}^{M}
\end{array}\right\}=\left[\begin{array}{cc}
-\frac{1}{\rho_{j}} & \tau_{j}^{0} \frac{\partial}{\partial s_{j}} \\
\frac{\partial}{\partial s_{j}} & \tau_{j}^{0} \frac{1}{\rho_{j}}
\end{array}\right]\left\{\begin{array}{l}
\sigma^{s u r, j} \\
\omega^{s u r, j}
\end{array}\right\} \\
=\left[\begin{array}{cc}
-\frac{1}{\rho_{j}} & \tau_{j}^{0} \frac{\partial}{\partial s_{j}} \\
\frac{\partial}{\partial s_{j}} & \tau_{j}^{0} \frac{1}{\rho_{j}}
\end{array}\right]\left\{\begin{array}{c}
\tau_{j}^{0}+\left(2 \mu_{j}^{s}+\lambda_{j}^{s}\right) \varepsilon^{s u r, j} \\
\omega^{s u r, j}
\end{array}\right\} \\
\left\{\begin{array}{l}
\varepsilon^{s u r, j} \\
\omega^{s u r, j}
\end{array}\right\}=\left[\begin{array}{ll}
\frac{1}{\rho_{j}} & \frac{\partial}{\partial s_{j}} \\
\frac{\partial}{\partial s_{j}} & -\frac{1}{\rho_{j}}
\end{array}\right]\left\{\begin{array}{l}
u_{n}^{j} \\
u_{t}^{j}
\end{array}\right\}=\left[\begin{array}{ll}
\frac{1}{\rho_{j}} & \frac{\partial}{\partial s_{j}} \\
\frac{\partial}{\partial s_{j}} & -\frac{1}{\rho_{j}}
\end{array}\right]\left[\begin{array}{cc}
n_{1} & n_{2} \\
-n_{2} & n_{1}
\end{array}\right]\left\{\begin{array}{l}
u_{1}^{j} \\
u_{2}^{j}
\end{array}\right\}
\end{gathered}
$$


Following substitution of Eq. (5) into Eq. (4), the interface equilibrium conditions can be recast in the following form:

$$
\begin{aligned}
& \left\{\begin{array}{l}
\sigma_{n}^{j}-\sigma_{n}^{M} \\
\sigma_{t}^{j}-\sigma_{t}^{M}
\end{array}\right\}=\left\{\begin{array}{c}
-\frac{1}{\rho_{j}} \\
\frac{\partial}{\partial s_{j}}
\end{array}\right\} \tau_{j}^{0} \\
& +\left[\begin{array}{l}
\left.\left.-\alpha_{j} \frac{1}{\left(\rho_{j}\right)^{2}}+\tau_{j}^{0} \frac{\partial^{2}}{\partial s_{j}^{2}}\right) \quad\left(\frac{-\alpha_{j}}{\rho_{j}} \frac{\partial}{\partial s_{j}}-\left(\frac{\tau_{j}^{0}}{\rho_{j}} \frac{\partial}{\partial s_{j}}-\frac{\tau_{j}^{0}}{\rho_{j}^{2}} \frac{\partial \rho_{j}}{\partial s_{j}}\right)\right)\right]\left[\begin{array}{ll}
n_{1} & n_{2} \\
-n_{2} & n_{1}
\end{array}\right]\left\{\begin{array}{l}
u_{1}^{j} \\
u_{2}^{j}
\end{array}\right\} \\
{\left[\left(\frac{\alpha_{j}}{\rho_{j}} \frac{\partial}{\partial s_{j}}-\frac{\alpha_{j}}{\rho_{j}^{2}} \frac{\partial \rho_{j}}{\partial s_{j}}\right)+\frac{\tau_{j}^{0}}{\rho_{j}} \frac{\partial}{\partial s_{j}}\right)}
\end{array}\right.
\end{aligned}
$$

In the above, we have that $\alpha_{j}=\left(2 \mu_{j}^{S}+\lambda_{j}^{S}\right)$ is a material parameter, $n_{1}=n_{1}^{j}=-n_{1}^{M} ; n_{2}=n_{2}^{j}=-n_{2}^{M}$ are the components of the normal vector as viewed from either side of an interface, $\rho_{j}$ is the curvature radius of the $j$-th interface $\Gamma_{i}^{j}$, and subscripts $t$ and $n$ denote the tangential (counter-clockwise is positive) and normal directions along $\Gamma_{i}^{j}$. Furthermore, $\varepsilon^{\text {sur }}, \omega^{\text {sur }}$ respectively are the strain and rotation vectors that develop at the surface. Finally, in the case of a nano-cavity, the normal and tangential stresses are $\sigma_{n}^{j}=\sigma_{t}^{j}=0$.

In so far as the direct BEM formulation with displacements and tractions as the field variables is concerned, the boundary condition in Eq. (6) can be expressed in terms of tractions alone, bypassing the need to introduce normal and tangential displacement components $u_{n}^{j}$ and $u_{t}^{j}$ as follows:

$$
\left\{\begin{array}{c}
t_{1}^{j}+t_{1}^{M} \\
t_{2}^{j}+t_{2}^{M}
\end{array}\right\}=\left\{\mathbf{f}^{s u r}\right\}+\left[\mathbf{T}^{s u r}\right]\left\{\begin{array}{l}
u_{1}^{j} \\
u_{2}^{j}
\end{array}\right\}
$$

In the above reformulation, we have the surface force vector and the surface traction matrix respectively defined as follows:

$$
\begin{aligned}
& \left\{\mathbf{f}^{\text {sur }}\right\}=-\frac{\tau_{j}^{0}}{\rho_{j}}\left\{\begin{array}{l}
n_{1} \\
n_{2}
\end{array}\right\} \\
& {\left[\mathbf{T}^{s u r}\right]=\left[\begin{array}{cc}
n_{1} & -n_{2} \\
n_{2} & n_{1}
\end{array}\right]\left[\begin{array}{cc}
\left(-\alpha_{j} \frac{1}{\left(\rho_{j}\right)^{2}}+\tau_{j}^{0} \frac{\partial^{2}}{\partial s_{j}^{2}}\right) & \left(\frac{-\alpha_{j}}{\rho_{j}} \frac{\partial}{\partial s_{j}}-\left(\frac{\tau_{j}^{0}}{\rho_{j}} \frac{\partial}{\partial s_{j}}-\frac{\tau_{j}^{0}}{\rho_{j}^{2}} \frac{\partial \rho_{j}}{\partial s_{j}}\right)\right) \\
\left(\left(\frac{\alpha_{j}}{\rho_{j}} \frac{\partial}{\partial s_{j}}-\frac{\alpha_{j}}{\rho_{j}^{2}} \frac{\partial \rho_{j}}{\partial s_{j}}\right)+\frac{\tau_{j}^{0}}{\rho_{j}} \frac{\partial}{\partial s_{j}}\right) & \left(\alpha_{j} \frac{\partial^{2}}{\partial s_{j}^{2}}-\tau_{j}^{0} \frac{1}{\left(\rho_{j}\right)^{2}}\right)
\end{array}\right]\left[\begin{array}{cc}
n_{1} & n_{2} \\
-n_{2} & n_{1}
\end{array}\right]}
\end{aligned}
$$

The solution of the BVP formulated above satisfies the governing equations, Eq. (1), plus the continuity (or essential) boundary conditions of Eq. (3) and the 
equilibrium (or natural) boundary conditions of Eq. (7) along each interface $\Gamma_{i}^{j}$, where $j=1,2, \ldots N$. Note that when the membrane interface parameters $\tau_{j}^{0}=0$, $\lambda_{j}^{S}=\mu_{j}^{S}=0$, then boundary condition of Eq. (6) transforms into the classical boundary condition for traction continuity.

\section{BEM development and numerical implementation}

The BEM statement for time-harmonic elastodynamics is used here in the form

$$
c_{i j} u_{j}^{s c}(\mathbf{x}, \omega)=\int_{S} U_{i j}^{*}(\mathbf{x}, \mathbf{y}, \omega) t_{j}^{s c}(\mathbf{y}, \omega) d S(\mathbf{y})-\int_{S} P_{i j}^{*}(\mathbf{x}, \mathbf{y}, \omega) u_{j}^{s c}(\mathbf{y}, \omega) d S(\mathbf{y}), \mathbf{x} \in S
$$

In the above, $c_{i j}$ are jump terms dependent on the local geometry at the collocation point $\mathbf{x}\left(x_{1}, x_{2}\right)$, surface $S=\Gamma_{I}$, and $U_{i j}^{*}$ is the displacement fundamental solution of the governing equation (1). Also, $P_{i j}^{*}=C_{i j q l} U_{q k, l}^{*} n_{k}$ is the corresponding traction fundamental solution. The unknowns of the problem are now the scattered wave field displacements along all interface boundaries. The displacements and stresses at any point in the surrounding elastic matrix can be obtained from the well-known integral representation formulas using the solution of Eq. (9) for displacements and tractions along all interface boundaries.

The numerical implementation of the above boundary integral equations follows standard procedure, in the sense that all boundaries of the problem in question (in our case this would be all interfaces $\Gamma_{i}^{j}, j=1,2 \ldots \mathrm{N}$ ) are discretized into segments using three-node, quadratic boundary elements (BE). We also distinguish between the weakly singular displacement kernel that can be numerically integrated by special-purpose Gaussian quadrature, supplemented by a breakdown of the singular BE into sub-elements for better accuracy, and the strongly singular traction kernel that is integrated indirectly by applying the rigid-body motion concept. Next, nodal collocation leads to a system of linear algebraic equations. We note that for wave propagation problems, the incoming elastic wave carries a stress field that can be converted to input tractions at all surfaces. Furthermore, for the purposes of this work, the BEM formulation is enhanced by sub-structuring capabilities, whereby it is possible to model heterogeneities and the surrounding elastic matrix separately as if they were independent structures, and reconstituting the complete solution through application of the boundary conditions. Finally, the present BEM implementation was carried out using the MATLAB [12] software package.

\section{Numerical verification studies}

Verification of the above BEM development is accomplished by running a number of test examples for both static and time-harmonic loading conditions. 


\subsection{Test example 1: in-plane nano-scale circular cavity under a static load}

Consider an infinite, linear elastic plane with a single, nano-scale cylindrical hole of radius $a_{0}=2.10^{-9} \mathrm{~m}$. The plane is under a far-field loading and the surface stresses acting at the hole boundary are modeled as a thin film with different values for the surface elasticity parameters $\lambda^{S}$ and $\mu^{S}$, keeping the elastic properties of the surrounding medium fixed as $\lambda_{M}=60 \mathrm{GPa}, \mu_{M}=26.13 \mathrm{GPa}$. Plane strain condition are assumed, and two far-field loads are considered: (a) Uniform tension along axis $O x_{2}$, i.e. $t_{1}=0 ; t_{2}=\sigma_{0}$; (b) bi-axial loading, i.e. $t_{1}=0.5 \sigma_{0} ; t_{2}=\sigma_{0}$. We introduce parameter $M_{S}=2 \mu^{S}+\lambda^{S}-\tau^{0}$, and the special case of $\tau^{0}=0$ is used for validation purposes. This test example was solved analytically in Tian and Rajapakse [4] and in Grekov and Morozov [13], who reduced the BVP to a hypersingular integral equation with respect to the unknown surface stress by applying the Goursat-Kolosov's complex potentials and Muskhelishvili's technique, respectively. A cylindrical coordinate system $\left(r, \varphi, x_{3}\right)$ with the $x_{3}$ axis along the diameter of the cylindrical inclusion is adopted for this problem. Figure 1 shows a comparison between the authors' BEM results and analytical solution obtained in [13] for the variation of the normalized stress $\sigma_{\varphi \varphi} / \sigma_{0}$ ratio along the perimeter of the circular hole with fixed radius $a_{0}$ and for different values for the elastic properties of the interface boundary parameter $M_{S}$ (in $N / m$ ). The BEM mesh employed here comprised 32 quadratic (three-node) BE. The solution, as obtained by the authors, reproduces the analytical solution derived in Grekov and Morozov [13] exactly.

(a)

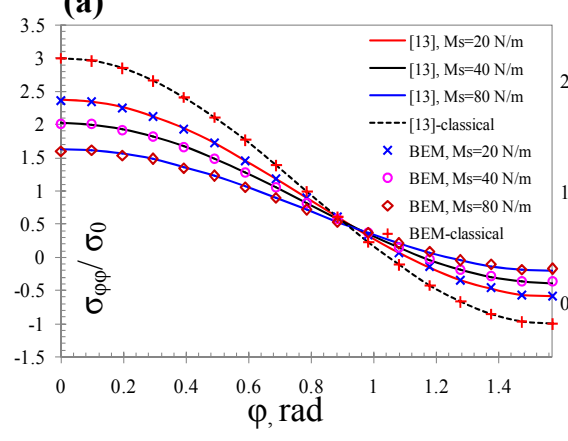

(b)

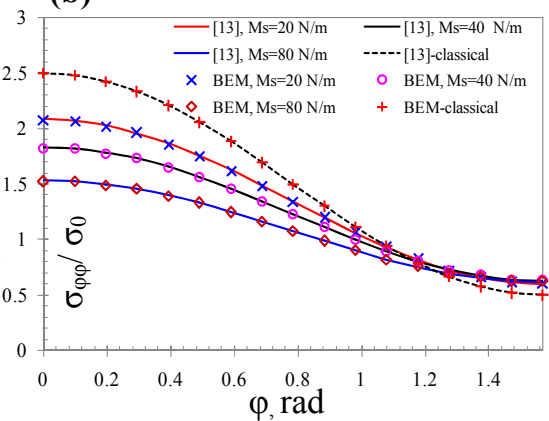

Figure 1: $\quad$ Stress ratio $\sigma_{\varphi \varphi} / \sigma_{0}$ along the circular hole perimeter for different surface elastic properties Ms: (a) uniform tension; (b) biaxial loading. 


\subsection{Test example 2: in-plane nano-scale circular inclusion under a P-wave}

An infinite solid matrix with material constants $\lambda_{M}, \mu_{M}$, and $\rho_{M}$ contains an elastic cylindrical nano-inclusion with radius $a_{0}$ and material properties $\lambda_{I}, \mu_{I}$, and $\rho_{I}$. The surface elastic properties are represented by the dimensionless parameter $s=M_{S} / 2 a_{0} \mu_{M}$. Assume as the excitation a time-harmonic P-wave with frequency $\omega$ propagating along the positive $O x_{1}$ direction. When the wave first impinges on the inclusion-matrix interface, a reflected wave is generated that propagates back into the matrix, while a refracted wave moves into the inclusion. We define a non-dimensional frequency with respect to the solid matrix material properties as $\Omega_{M}=\omega a_{0} / C_{P}^{M}$. Consider now the case with fixed ratios of $\mu_{I} / \mu_{M}=0.2$ and $\Omega_{I} / \Omega_{M}=3.0$, plus a constant value of Poisson ratio's for both solid phases as $v=0.26$. Figure 2 presents a comparison between the authors' $\mathrm{BEM}$ solution and the results obtained by $\mathrm{Ru}$ et al. [7] for the dynamic stress concentration factor (DSCF) defined as $\left|\sigma_{\varphi \varphi} / \sigma_{0}\right|$ along the interface boundary, where $\sigma_{0}=-\mu_{M} \beta^{2} u_{0}, \beta=\omega / C_{S V}$ and $C_{S V}=\sqrt{\mu_{M} / \rho_{M}}$. We consider a range of values for parameter $s$ and two values for the frequencies as $\Omega_{M}=0.2$ and $\Omega_{M}=\pi$. All results to within plotting accuracy, despite having been generated by two different computational techniques. We note here that $\mathrm{Ru}$ et al. [7] used the displacement potential method. The BEM mesh employed comprised $24 \mathrm{BE}$ for $\Omega_{M}=0.2$ and $64 \mathrm{BE}$ for $\Omega_{M}=\pi$.
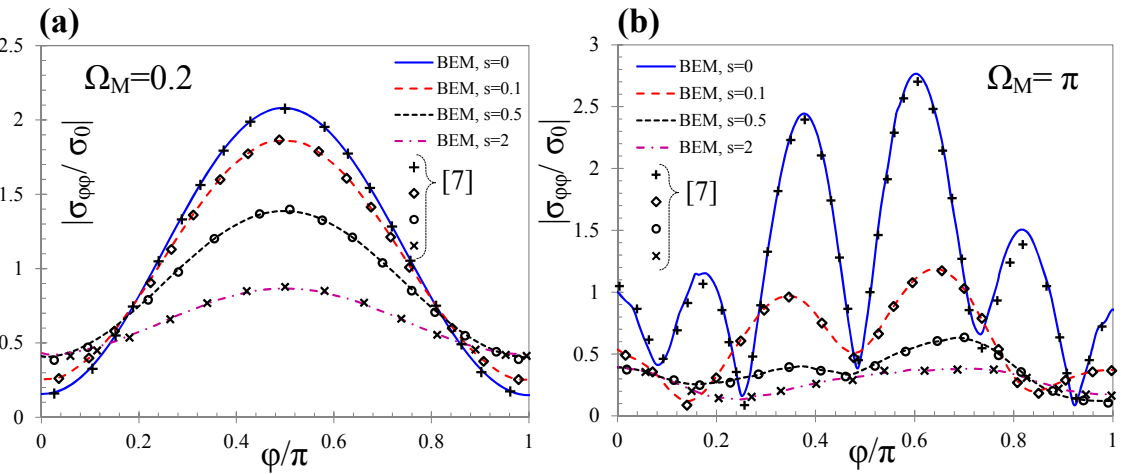

Figure 2: $\quad$ Stress ratio $\left|\sigma_{\varphi \varphi} / \sigma_{0}\right|$ along the interface for case $\mu_{I} / \mu_{M}=0.2$, $\Omega_{I} / \Omega_{M}=3.0$ and at frequency values of: (a) $\Omega \mathrm{M}=0.2$; (b) $\Omega \mathrm{M}=\pi$. 


\subsection{Test example 3: two in plane nano-scale circular holes under a P-wave}

Two circular cylindrical holes of equal radius $a$ and center-to-center separation distance $2 b$ (see Figure 3) are placed in the infinite plane with material properties $\lambda_{M}, \mu_{M}$, and $\rho_{M}$. As before, a time-harmonic P-wave propagates through the solid matrix with frequency $\omega$ and incident angle $\theta=0$ with respect to the horizontal axis. The surface elastic properties are described by the dimensionless parameter $s=M_{S} / 2 a \mu_{M}$, while Poisson's ratio for the solid medium is $v=0.26$.

The following notations are introduced: $\Omega=\omega a / C_{P}, \mathrm{DSCF}=\left|\sigma_{\varphi_{1} \varphi_{1}} / \sigma_{0}\right|$.

This test example was solved analytically by Wang [5], who used the wave function expansion method and the translational addition theorem. In Figure 3 we compare our BEM results with those of Wang [5]. Our BEM mesh comprised 32 quadratic BE.

(a)

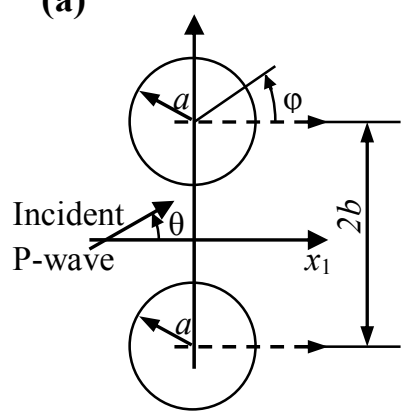

(b)

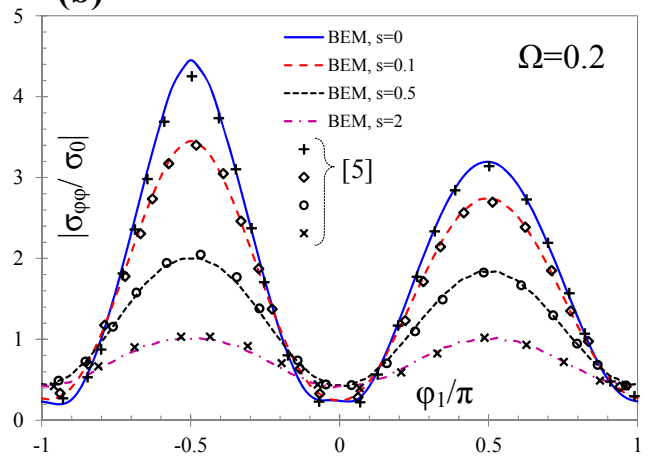

Figure 3: (a) Geometry; (b) DSCF along the upper circular hole perimeter at various s values for $\Omega=0.2$ and at $\mathrm{b} / \mathrm{a}=1.25$.

All figures in this section conclusively demonstrate the accuracy and convergence of the BEM developed in this work for the solution of the general elastic wave diffraction and scattering problem by nano-inhomogeneities placed within an infinite solid matrix.

\section{Parametric studies}

The aim here is to solve the problem involving multiple nano-cavities or nano-inclusions in an elastic matrix of infinite extent, so as to investigate the sensitivity of the DSCF that develops at the interfaces to the following key factors: (a) surface properties, as quantified by the interfacial material constants; (b) type of the nano-heterogeneity; (c) dynamic interactions between multiple nanocavities and/or nano-inclusions, including their number and geometrical configuration; (d) frequency content and incidence angle of the incoming wave. 

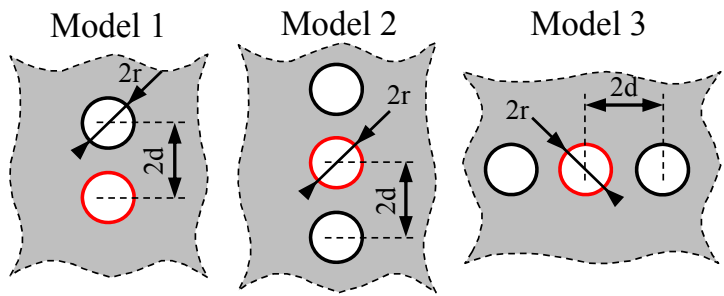

Model 4

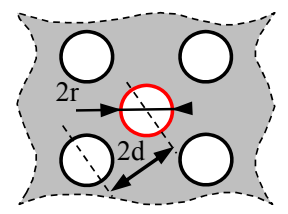

Figure 4: Geometry of the four models considered in the BEM simulations.

We consider the four models of Figure 4 to study multiple scattering and diffraction phenomena in an infinite elastic matrix with nano-heterogeneities in the form of either cavities or elastic inclusions. The heterogeneities have circular shape with radius $r=1 \mathrm{~nm}$ and the center-to-center separation distance of $2 d=2.5 \mathrm{~nm}$. The material properties of the infinite plane are $\lambda_{M}, \mu_{M}, \rho_{M}$ in consistent units, while those of the inclusions are $\lambda_{I}, \mu_{I}, \rho_{I}$, Poisson ratio is $v=0.26$ for both phases. For the case of the inclusions, we define the ratios $\mu_{I} / \mu_{M}=0.2$ and $\Omega_{I} / \Omega_{M}=3.0$. In terms of results, the DSCF along the perimeter of the central hole is plotted in Figure 5 and that of the inclusion in Figure 6, for different values of the dimensionless parameter $s$ and fixed nondimensional frequency $\Omega=\omega r / C_{P}^{M}$ of the P-wave with incident angle $\theta=0$.
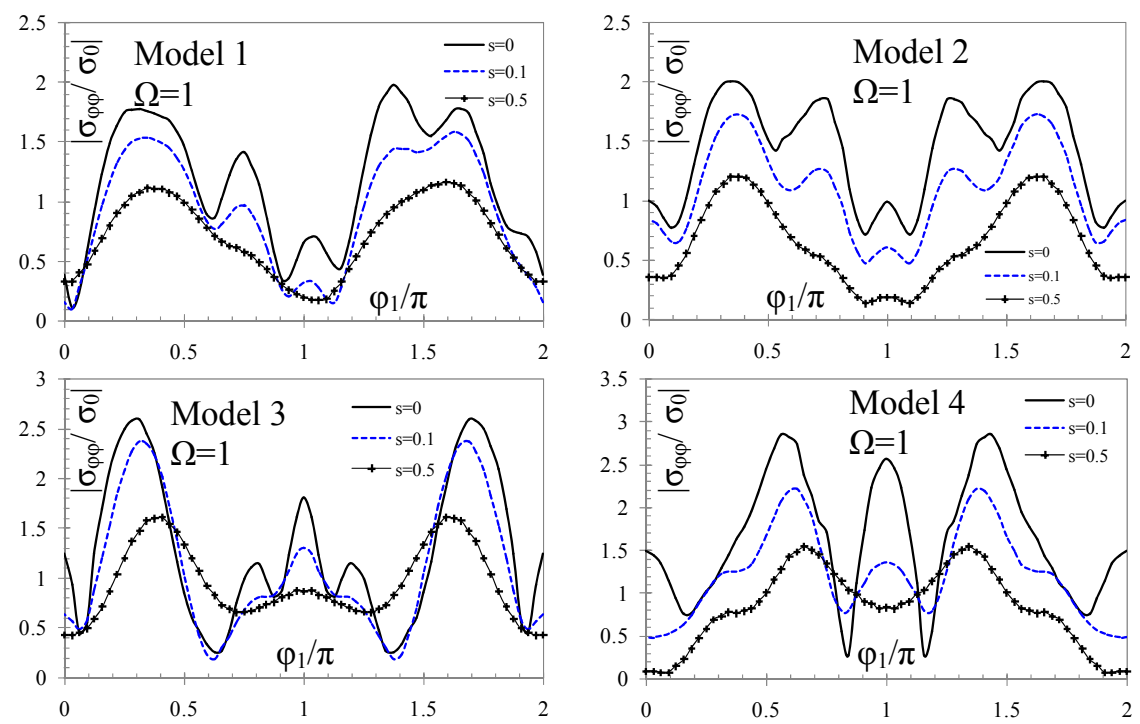

Figure 5: $\quad$ DSCF along the central circular cavity perimeter at fixed $\Omega$ and for different values of the interface surface parameter $\mathrm{s}$. 
The following conclusions can be drawn based on the above numerical simulations: (a) the surface effects and the dynamic interaction between multiple heterogeneities, regardless of cavities or elastic inclusions, clearly show multiple wave scattering and diffraction phenomena; (b) the ensuing DSCF is sensitive to the geometrical configuration, size and type of nano-heterogeneities, wave frequency and incident angle, elastic properties of the matrix and of the inclusions, plus surface properties of the interfaces; (c) independently of the incident wave frequency and of the distance and configuration between the multiple cavities or the elastic inclusions, surface elasticity always tends to depress the DSCF values which develop; (d) a key factor for this problem seems to be the ratio of the incident wave length to the center-to-center separation distance, which heavily influences on the non-uniform stress distribution in the infinite matrix.
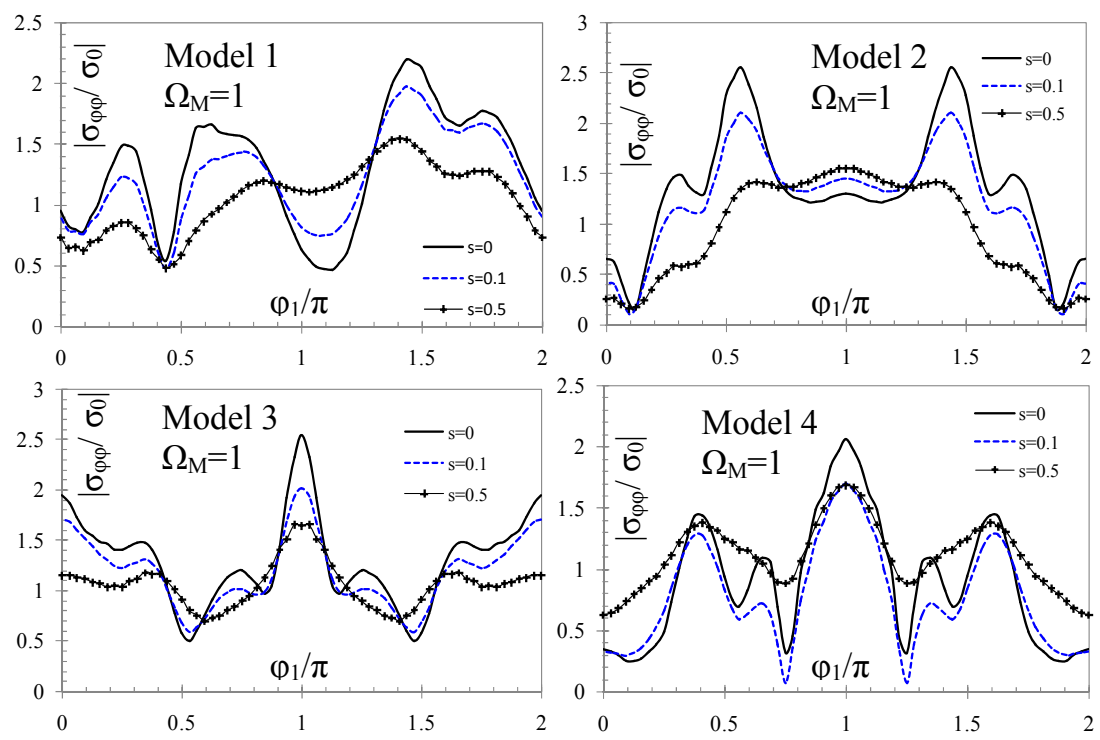

Figure 6: DSCF along the central circular inclusion perimeter at fixed $\Omega \mathrm{M}$ and for different values of the interface surface parameter $s$.

\section{Conclusions}

In this work, the two-dimensional elastodynamic problem of in-plane motion is solved in the frequency domain for a heterogeneous elastic matrix containing multiple nano-heterogeneities. The computational approach developed for this purpose is the direct BEM, employing fundamental solutions that solve the NavierCauchy equations of dynamic equilibrium for the bulk solid. This BEM formulation is augmented by the surface elasticity model of Gurtin and Murdoch [2], which accounts for the proper boundary conditions at the free surfaces and the interfaces when the solution sought at the nano-level for the heterogeneities. Next, 
this augmented BEM is successfully gauge against a number of benchmark examples and is subsequently used to compute the DSCF around the nano heterogeneities embedded in the solid matrix.

The novelty of the present numerical approach lies in the effective combination of four stages, namely (a) mechanical modeling based on classical elastodynamic theory for the bulk solid, (b) introduction of non-classical boundary conditions stemming from localized constitutive equations for the material interfaces, (c) verification of an efficient BEM-based computational tool and (d) extensive numerical simulations for the benchmark problem of an elastic matrix containing multiple nano-heterogeneities that act as both wave diffractors and stress concentrators. In sum, the present work serves as a valuable tool in the effort to develop new classes of nano-components and nano-structures for the electronic industry, for optics, for biomedical engineering purposes, and for other novel technological fields.

\section{Acknowledgement}

The first author (SLP) wishes to acknowledge the support provided through a Research and Design Centre of UACEG, Sofia grant No. BN 158/14.

\section{References}

[1] Srivastava, D., Atluri S.N., Computational Nanotechnology: A Current Perspective. Computer Modeling in Engineering and Sciences, 3(5), pp. 531-538, 2002.

[2] Gurtin, M.E., Murdoch, A.I., A continuum theory of elastic material surfaces. Archives Rational Mech. Anal., 57, pp. 291-323, 1975.

[3] Gurtin, M.E., Murdoch, A.I., Surface stress in solids. Int. J. Solids Structures, 14, pp. 431-440, 1978.

[4] Tian, L., Rajapakse, R.K.N.D., Analytical solution for size-dependent elastic field of a nano-scale circular inhomogeneity. Journal of Applied Mechanics ASME, 74, pp. 568-574, 2007.

[5] Wang, G.F., Multiple diffraction of plane compressional waves by two circular cylindrical holes with surface effect. Journal of Applied Physics, 105, pp. 013507-1-013507-6, 2009.

[6] Wang, W., Zeng, W., Ding, J., Finite element modeling of two-dimensional nanoscale structures with surface effects. World Academy of Science, Engineering and Technology, 46, pp. 12-20, 2010.

[7] Ru, Y., Wang, G.F., Wang, T.J., Diffraction of elastic waves and stress concentration near a cylindrical nano-inclusion incorporating surface effect. Journal of Vibration and Acoustics, 13, pp. 061011-1-061011-7, 2009.

[8] Dong, C.Y., An integral equation formulation of two- and threedimensional nanoscale inhomogeneities. Computational Mechanics, 49, pp. 309-318, 2012. 
[9] Jammes, M., Mogilevskaya, S.G., Crouch, S.L., Multiple circular nano-inhomogeneities and/or nano-pores in one of two joined isotropic half-planes. Engineering Analysis with BE, 33, pp. 233-248, 2009.

[10] Gurtin, M.E., Weissmuller, J., Larche, F., A general theory of curved deformable interfaces in solids at equilibrium. Philosophical Magazine, 78, pp. 1093-1109, 1998.

[11] Dong, C.Y., Pan, E., Boundary element analysis of nano-inhomogeneities of arbitrary shapes with surface and interface effects. Engineering Analysis with BE, 35, pp. 996-1002, 2011.

[12] MATLAB The Language of Technical Computing, Version 7.9. The MathWorks, Inc., Natick, Massachusetts, 2009.

[13] Grekov, M.A., Morozov, N., Surface effects and problems of nanomechanics. Journal of Ningbo University, 25(1), pp. 60-63, 2012. 